\title{
Characterization of Dentine, Dentinal Tubules and Dentine-Enamel Junction in Human Teeth by Advanced Analytical TEM
}

\author{
V. Srot ${ }^{*}$, B. Bussmann*, U. Salzberger ${ }^{*}$, C.T. Koch ${ }^{*}$, G. Čižmek ${ }^{* *}$, and P.A. van Aken ${ }^{*}$ \\ * Stuttgart Center for Electron Microscopy, Max Planck Institute for Metals Research, Stuttgart, \\ Germany \\ ${ }^{* *}$ Medical Center Velenje, Velenje, Slovenia
}

A wide range of organisms produce a great variety of biominerals. Many of these biominerals are composite or agglomerated materials, where an organic matrix and amorphous or crystalline minerals are linked together [1]. These extraordinary combinations with unique and complex architectures lead to highly improved and advanced materials characteristics compared to their inorganic mineralogical counterparts [2]. This is because organism produced and controlled materials have unique microstructures, since they are synthesized at ambient temperatures and atmospheric conditions [3].

Two fundamental classes of calcium $(\mathrm{Ca})$ bearing minerals, $\mathrm{Ca}$ carbonates and $\mathrm{Ca}$ phosphates, are widespread constituents of teeth, bones and shells. They are thermodynamically stable within biological environment due to their high lattice energies and low solubility [3]. Human mineralized tissues, such as teeth and bones, consist of inorganic $\mathrm{Ca}$ phosphate mineral in the form of hydroxylapatite and an organic component, mainly collagen. Biological hydroxylapatite is structurally and chemically very complex and it could be described as an impure Ca-deficient carbonate-containing apatite involving a variety of other minor constituent ions [3].

Dentine is a mineralized tissue that forms the bulk of the teeth. In the crown dentine is protected by enamel and in the root it is covered by cementum. Dentine is composed of approximately $70 \mathrm{wt} \%$ of inorganic material, $20 \mathrm{wt} \%$ of organic material, and $10 \mathrm{wt} \%$ of water. Numerous dentinal tubules penetrate the dentine and they extend from the pulp towards the dentine-enamel or dentine-cement junctions [4]. The dentinal tubules are surrounded by a wall of highly mineralized peritubular dentin and are separated by intertubular dentin [5].

For our investigations, milk and permanent human teeth were used. Detailed studies of dentine and dentinal tubules from different locations in the teeth were performed by using energy-dispersive Xray spectroscopy (XEDS) and electron energy-loss spectroscopy (EELS). P-L $\mathrm{L}_{2,3}, \mathrm{C}-\mathrm{K}, \mathrm{Ca}-\mathrm{L}_{2,3}$, N-K and $\mathrm{O}-\mathrm{K}$ electron energy-loss near-edge structures (ELNES) were acquired at high energy and high spatial resolution using the Zeiss SESAM and VG HB501UX microscopes.

A bright field (BF) scanning transmission electron microscopy (STEM) image of the dentinal tubules (DT) is shown in Figure 1a. An image at higher magnification (Fig. 1b) clearly shows a well mineralized wall of dentinal tubule consisting of peritubular dentin (PD) and an intertubular dentin (ID) which represents the main body of dentine being located between the dentinal tubules. According to our XEDS measurements there is a higher content of magnesium in PD compared to ID, as already shown by Lefèvre et al. [6] by using secondary ion mass spectrometry. Therefore also EELS experiments were performed in order to measure possible differences. $\mathrm{Ca}-\mathrm{L}_{2,3}$ edges acquired from ID and PD are presented in Figure 2. C-K ELNES from ID and PT are different, most probably due to different amount of organic material (Fig. 2a). The presence of nitrogen originates from collagen and is therefore more pronounced in spectra acquired from ID (c.f. N-K edge in the upper spectrum of Fig. 2a). There is no noticeable difference in the number and position of peaks in the Ca-L ${ }_{2,3}$ ELNES between PD and ID (Fig. 2b). 
Detailed knowledge of microstructure, properties and chemistry of dentine are of great importance, since they have important consequences for different fields of dentistry. Improved understanding of the dentinal tubules is crucial, since they are responsible for the permeability of dentine [7]. Presented results enhance the understanding of differences in microstructure and chemical composition of dentine and dentinal tubules. Additionally, also possible differences in chemical composition in the vicinity of the dentine-enamel junction were studied and will be discussed.

\section{References}

[1] P.U.P.A. Gilbert et al., Reviews in Mineralogy \& Geochemistr, 59 (2005) 157.

[2] A. P. Jackson et al., J. Mat. Sci., 25 (1990) 3173.

[3] S. Mann, Biomineralization, Oxford University Press, New York, 2001.

[4] B.K.B. Berkovitz et al., Teeth, Springer-Verlag, Berlin, 1989.

[5] F.H. Jones, Surface Science Reports, 42 (2001) 75.

[6] Lefèvre et al., Calcif. Tiss. Res., 19 (1976) 251.

[7] I.A. Mjör et al., Archs. Oral Biol., 41 (1996) 401.
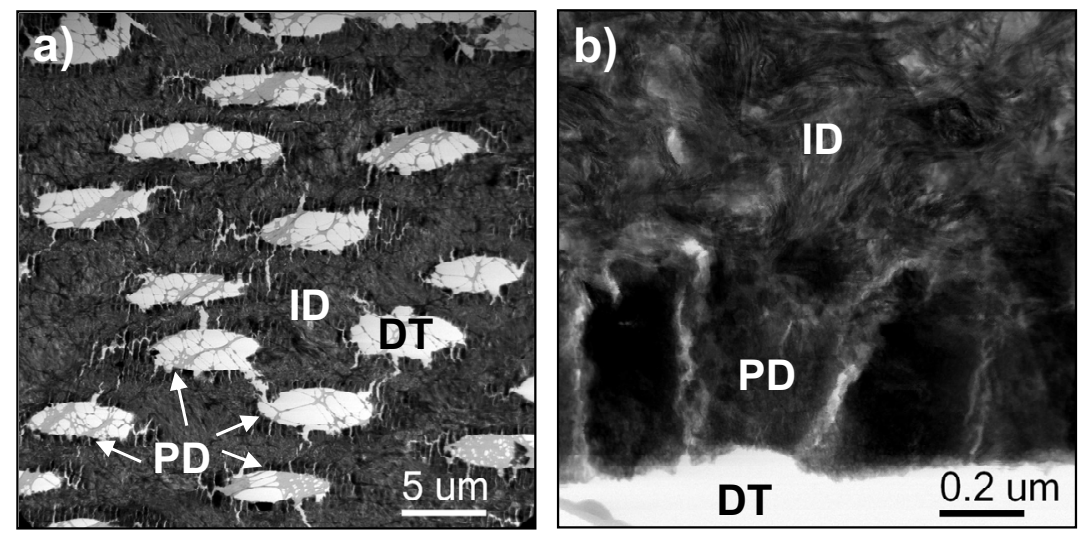

FIG. 1: (a) BF-STEM images of dentinal tubules (DT) separated by intertubular dentin (ID) and surrounded by peritubular dentine (PD). In (b) an enlarged area is presented.
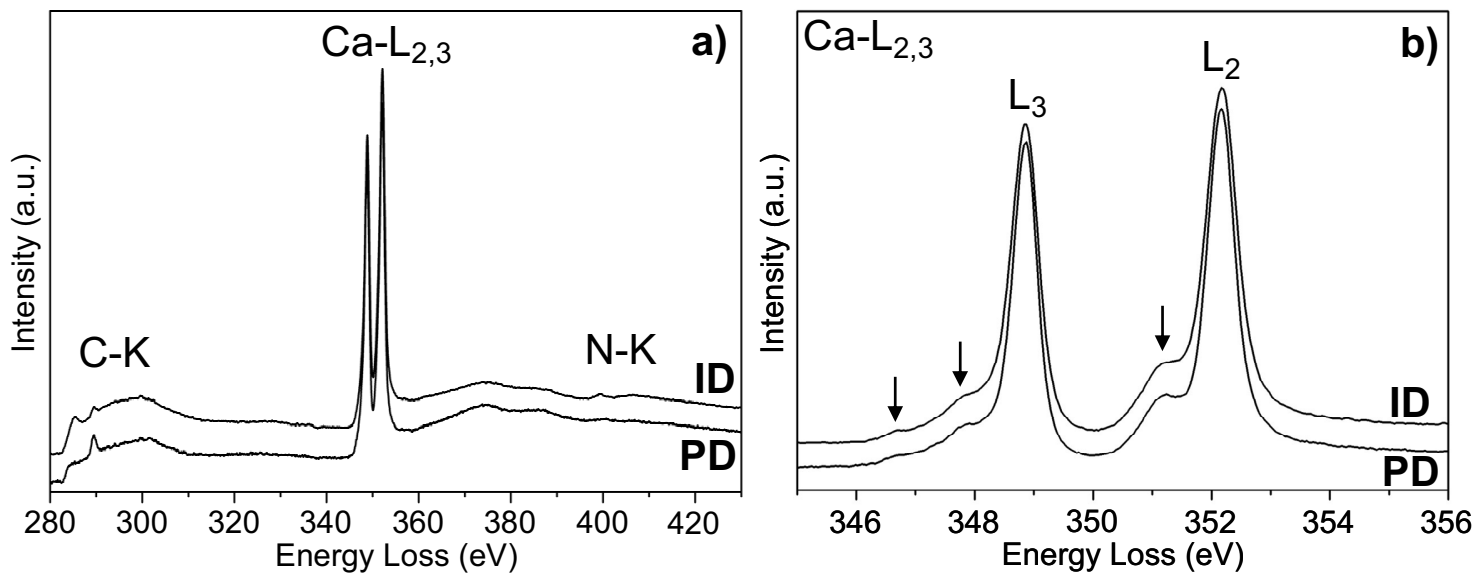

FIG. 2: ELNES acquired in the energy range between 280 and $430 \mathrm{eV}$ from peritubular (PD) and intertubular (ID) dentin showing $\mathrm{C}-\mathrm{K}, \mathrm{Ca}-\mathrm{L}_{2,3}$ and $\mathrm{N}-\mathrm{K}$ edges in (a). In (b) $\mathrm{Ca}-\mathrm{L}_{2,3}$ ELNES from peritubular (PD) and intertubular (ID) dentine with marked fine spectral features are shown. 